\title{
Raquianestesia para Cesariana com Bupivacaína a 0,5\% Isobárica Associada ao Fentanil e Morfina. Estudo Prospectivo com Diferentes Volumes *
}

\section{Spinal Anesthesia for Cesarean Section with 0.5\% Isobaric Bupivacaine plus Fentanyl and Morphine. Prospective Study with Different Volumes}

Luiz Eduardo Imbelloni, TSA ${ }^{1}$; Eneida Maria Vieira ${ }^{2}$; Ana Rocha ${ }^{3}$; Marildo Assunção Gouveia, TSA ${ }^{4}$; José Antônio Cordeiro ${ }^{5}$

\begin{abstract}
RESUMO
Imbelloni LE, Vieira EM, Rocha A, Gouveia MA, Cordeiro JA Raquianestesia para Cesariana com Bupivacaína a 0,5\% Isobárica Associada ao Fentanil e Morfina. Estudo Prospectivo com Diferentes Volumes
\end{abstract}

JUSTIFICATIVA E OBJETIVOS: A raquianestesia para cesariana foi descrita poucos anos após o primeiro relato de Bier em 1898 e nos últimos 5 anos ela se tornou método de escolha em nosso hospital. O objetivo deste estudo prospectivo em cesariana é avaliar o uso da bupivacaína a $0,5 \%$ isobárica, administrada com as parturientes em decúbito lateral, após injeção de fentanil e morfina, e correlacionar com a incidência de complicações hemodinâmicas e dispersão cefálica com diferentes volumes.

MÉTODO: Cem pacientes submetidas à raquianestesia para cesariana eletiva foram aleatoriamente separadas em três grupos que receberam: $4 \mathrm{ml}(20 \mathrm{mg}), 3 \mathrm{ml}(15 \mathrm{mg})$ e 2, $5 \mathrm{ml}(12,5$ $\mathrm{mg}$ ) de bupivacaína a 0,5\% isobárica acrescida de $25 \mu \mathrm{g}$ de fentanil e $50 \mu \mathrm{g}$ de morfina injetadas antes do anestésico. Foram avaliados e comparados os seguintes parâmetros: latência da analgesia, bloqueio motor, dispersão cefálica da analgesia, alterações cardiovasculares e incidência de náuseas e vômitos.

RESULTADOS: Os três volumes de bupivacaina a 0,5\% isobárica produziram efeitos comparáveis. O tempo de latência foi maior com a menor dose. Não foram observadas diferenças na dispersão cefálica, no número de pacientes que tiveram níveis cervicais, nas alterações cardiovasculares e na incidência de cefaléia pós-punção. O nível máximo da analgesia foi $T_{4}$ (amplitude: $T_{3}-T_{6}$ ) com $4 \mathrm{ml}, T_{4}$ (amplitude: $T_{4}-T_{11}$ ) com $3 \mathrm{ml}$ e $T_{4}$ (amplitude: $T_{4}-T_{8}$ ) com 2,5 ml. Nenhuma paciente necessitou de efedrina para tratar hipotensão arterial. O

* Recebido do (Received from) CET/SBA Hospital de Base da Faculdade de Medicina de São José do Rio Preto, (FAMERP), São José do Rio Preto, SP

1. Anestesiologista da Casa de Saúde Santa Maria e Clínica São Bernardo. Rio de Janeiro, RJ

2. Auxiliar de Ensino na Faculdade de Medicina de São José do Rio Preto, $S P$

3. $M E_{2}$ do CET/SBA do Hospital de Base da FAMERP

4. Chefe do Serviço de Anestesiologia, Hospital Central do IASERJ, Rio de Janeiro, $R J$

5. Coordenador de Ensino na Faculdade de Medicina de São José do Rio Preto, SP

Endereço para correspondência (Correspondence to)

Dr. Luiz Eduardo Imbelloni

Av. Epitácio Pessoa, 2356/203, Lagoa

22471-000 Rio de Janeiro, $R J$

E-mail: imbelloni@openlink.com.br

Apresentado (Submitted) em 09 de abril de 2002

Aceito (Accepted) para publicação em 15 de outubro de 2002

(C) Sociedade Brasileira de Anestesiologia, 2003 bloqueio motor não foi completo em todas as pacientes. Uma paciente desenvolveu cefaléia pós-punção.

CONCLUSÕES: O resultado deste estudo confirma que a bupivacaína a 0,5\% isobárica injetada após administração, em seringas separadas de fentanil e morfina, e em decúbito lateral nos volumes de 2,5, 3 e $4 \mathrm{ml}$ proporciona uma rápida e efetiva anestesia para cesariana.

Unitermos: ANALGÉSICOS, Opióides: fentanil, morfina; ANESTÉSICOS, Local: bupivacaína; CIRURGIA, Obstétrica: cesariana; TÉCNICAS ANESTÉSICAS, Regional: subaracnóidea

\section{SUMMARY}

Imbelloni LE, Vieira EM, Rocha A, Gouveia MA, Cordeiro JA Spinal Anesthesia for Cesarean Section with $0.5 \%$ Isobaric Bupivacaine plus Fentanyl and Morphine. Prospective Study with Different Volumes

BACKGROUND AND OBJECTIVES: Spinal block for cesarean section was described few years after the first report of spinal anesthesia by Bier in 1899. It was not until the last 5 years that spinal anesthesia has become the most frequent anesthetic method for cesarean section at our hospital. This prospective study aimed at evaluating $0.5 \%$ spinal isobaric bupivacaine for cesarean section, injected after fentanyl and morphine, in the lateral position, and at correlating the incidence of hemodynamic changes and cephalad spread with different volumes.

METHODS: Participated in this study 100 patients undergoing spinal anesthesia for elective cesarean delivery who were randomly allocated into three groups to receive: $4 \mathrm{ml}(20 \mathrm{mg}), 3 \mathrm{ml}$ $(15 \mathrm{mg}$ ) or $2.5 \mathrm{ml}(12.5 \mathrm{mg})$ of $0.5 \%$ isobaric bupivacaine after $25 \mu \mathrm{g}$ fentanyl plus $50 \mu \mathrm{g}$ morphine. The following parameters were evaluated and compared: analgesia and motor block onset, cephalad spread of analgesia, cardiovascular changes and the incidence of nausea and vomiting.

RESULTS: The three volumes of $0.5 \%$ isobaric bupivacaine produced comparable effects. Onset was longer for the lowest dose. There were no differences in cephalad spread, number of patients with high cervical levels, cardiovascular changes and post dural puncture headache. Maximum analgesic level was $T_{4}$ (range: $T_{3}-T_{6}$ ) with $4 \mathrm{ml}, T_{4}$ (range: $T_{4}-T_{11}$ ) with $3 \mathrm{ml}$ and $T_{4}$ (range: $T_{4}-T_{8}$ ) with $2.5 \mathrm{ml}$. No patient required ephedrine to treat arterial hypotension. Motor block was incomplete for all patients. One patient developed post dural puncture headache. CONCLUSIONS: Results of this study confirm that $0.5 \%$ isobaric bupivacaine, following fentanyl and morphine injected with separate syringes and in the lateral position, in doses of 2.5, 3 and $4 \mathrm{ml}$ provides a fast and effective anesthesia for cesarean section.

Key Words: ANALGESICS, Opioids: fentanyl, morphine; ANESTHETICS, Local: bupivacaine; ANESTHETIC TECHNIQUES, Regional: spinal block; SURGERY, Obstetric: cesarean section 


\section{INTRODUÇÃO}

A raquianestesia vem sendo utilizada com mais freqüência do que a anestesia peridural para cesariana ${ }^{1}$, devido a algumas vantagens, como: identificação mais simples do espaço subaracnóideo, menor dose de anestésico local, menor custo e menor latência, com conseqüente menor tempo de permanência na sala de operações ${ }^{2-4}$.

Aintrodução de agulhas de fino calibre no início da década de $90 \mathrm{fez}$ ressurgir o interesse pela raquianestesia para todos os procedimentos compatíveis com a técnica. Araquianestesia para cesariana com bupivacaína a $0,5 \%$ hiperbárica cursa com uma incidência de hipotensão arterial de $37,5 \%{ }^{2}$ e o seu aparecimento é rápido ${ }^{2-4}$. Baseado em trabalho realizado em $1988^{5}$, a bupivacaína a $0,5 \%$ isobárica foi desaconseIhada para raquianestesia em obstetrícia, quando a punção fosse realizada na posição sentada ${ }^{6}$.

Na literatura nacional não existe publicação do emprego da bupivacaína a $0,5 \%$ isobárica em cesarianas. O objetivo deste estudo prospectivo é avaliar em cesarianas o uso da bupivacaína a $0,5 \%$ isobárica associada, em seringas separadas, com fentanil e morfina, administrada com as parturientes em decúbito lateral e correlacionar com a incidência de complicações hemodinâmicas e a dispersão cefálica com diferentes volumes.

\section{MÉTODO}

Após aprovação do Conselho de Ética do Hospital e consentimento formal, participaram deste estudo prospectivo 100 pacientes com idade igual ou superior a 15 anos e menor do que 40 anos, com peso entre 50 e $120 \mathrm{~kg}$ e estatura de $145 \mathrm{~cm}$ a $175 \mathrm{~cm}$, escaladas para cesarianas eletivas. Parturientes que apresentassem complicações obstétricas não foram incluídas no estudo. Critérios de exclusão foram hipovolemia, distúrbios de coagulação, infecção e recusa do método proposto. As pacientes foram aleatoriamente separadas em três grupos para receberem $4 \mathrm{ml}(20 \mathrm{mg}), 3 \mathrm{ml}$ (15 mg) ou 2,5 ml (12,5 mg) de bupivacaína a 0,5\% isobárica.

Nenhuma paciente recebeu medicação pré-anestésica no quarto. Após venóclise com cateter 18G, foi iniciada infusão de solução de Ringer com lactato. Não foi feita pré-expansão volumétrica e no final da cirurgia verificou-se o volume infundido.

Com a paciente em decúbito lateral esquerdo, a punção subaracnóidea foi realizada na linha mediana ou paramediana no espaço $L_{3}-L_{4}$, com agulha $27 \mathrm{G}$ tipo Quincke, sem auxílio de introdutor. Após refluxo de líquido cefalorraquidiano (LCR) através do canhão da agulha e com o bisel em sentido cefálico, foram previamente injetados $25 \mu \mathrm{g}$ de fentanil e 50 $\mu \mathrm{g}$ de morfina em seringas de insulina separadas e logo após o volume de bupivacaína isobárica a $0,5 \%$, de acordo com o grupo estudado, na velocidade de $1 \mathrm{ml} .15 \mathrm{seg}^{-1}$ sem barbotagem. Após a injeção as pacientes foram colocadas em decúbito dorsal horizontal, com o útero desviado para a esquerda. Freqüência cardíaca e pressão arterial materna foram avaliadas por método automático e não invasivo. Os valores ba- sais foram considerados os medidos no quarto. Os valores da pressão arterial e da freqüência cardíaca foram avaliados antes da punção subaracnóidea e depois, a cada três minutos, até 21 minutos e após cada cinco minutos, até o término da cirurgia. A saturação arterial do oxigênio foi continuamente monitorizada através de oximetria de pulso. O ECG foi monitorizado continuamente na posição CM5. A hipotensão arterial, determinada através da diminuição da PAS maior do que 30\% do valor da pressão anotado no quarto, seria tratada com 10 $\mathrm{mg}$ de efedrina, por via venosa. A freqüência cardíaca menor do que 60 bpm seria tratada com atropina, e náuseas e vômitos seriam tratadas com $20 \mathrm{mg}$ de metoclopramida por via venosa. Se ocorresse dor ou desconforto durante a cirurgia seriam administrados $50 \mu \mathrm{g}$ de fentanil e propofol em bolus. Oxigênio via cateter nasal foi administrado com um fluxo de 2 L. $\min ^{-1}$ do início até o final da cirurgia. Os neonatos foram avaliados pelo escore de Apgar no primeiro e quinto minutos de vida. Midazolam (1 a $3 \mathrm{mg}$ ) só foi administrado após a retirada do concepto. Foi anotado o tempo cirúrgico.

A latência do bloqueio foi definida como o tempo para a primeira perda da sensibilidade na região correspondente ao metâmero da punção. O nível segmentar da analgesia (perda da sensação à picada de agulha) foi determinada bilateralmente com intervalo de um minuto no início e a cada cinco minutos, até completar 20 minutos. Com o mesmo intervalo, foi pesquisado o grau de bloqueio motor dos membros inferiores, utilizando-se a escala modificada de Bromage.

Aqualidade do relaxamento muscular abdominal foi avaliada pelo cirurgião como excelente (com relaxamento muscular), satisfatório (aceitável relaxamento muscular) e insatisfatório (sem relaxamento muscular).

Foi utilizada cateterização vesical. Em todos as pacientes foi pesquisado o aparecimento de cefaléia até o quinto dia de pós-operatório.

Os dados estão expressos em média \pm DP, mediana ou freqüências (\%), quando apropriados. Os testes estatísticos utilizados foram teste Qui-quadrado com quadrados mínimos ponderados, teste Exato de Fisher, Análise de Variância (ANOVA), com comparações pareadas de Tukey, teste $t$ de Student univariado para dados pareados, sendo considerada diferença significante para $o$ valor de $p<0,05$.

\section{RESULTADOS}

Os dados demográficos e a população estudada estão na tabela I. Em virtude dos resultados obtidos com $3 \mathrm{ml}(15 \mathrm{mg}) \mathrm{e}$ $2,5 \mathrm{ml}$ (12,5 mg), o grupo com $4 \mathrm{ml}$ (20 mg) foi encerrado com 20 pacientes. Ocorreu uma falha da raquianestesia no grupo com $3 \mathrm{ml}$

Tabela I - Dados Demográficos (Média \pm DP)

\begin{tabular}{lcccc}
\hline & $\begin{array}{c}20 \mathrm{mg} \\
(\mathrm{n}=20)\end{array}$ & $\begin{array}{c}15 \mathrm{mg} \\
(\mathrm{n}=40)\end{array}$ & $\begin{array}{c}12,5 \mathrm{mg} \\
(\mathrm{n}=40)\end{array}$ & Valor de $\mathrm{p}$ \\
\hline Idade (anos) & $27,10 \pm 7,27$ & $24,75 \pm 6,13$ & $26,82 \pm 6,02$ & 0,25 \\
Peso (kg) & $68,25 \pm 8,46$ & $74,55 \pm 10,98$ & $75,97 \pm 14,03$ & 0,06 \\
Altura (cm) & $157,90 \pm 5,29$ & $162,20 \pm 6,17$ & $162 \pm 6,11$ & $0,02^{*}$ \\
\hline${ }^{*} \mathrm{p}<0,05$ & & & &
\end{tabular}

${ }^{*} p<0,05$ 
O início da analgesia foi rápido com as doses administradas. O tempo de latência foi significativamente maior com a menor dose (Tabela II). A dispersão cefálica da analgesia está na tabela II. Abaixa freqüência de nível sensitivo abaixo de $T_{5}$ faz com que uma análise estatística possa ser feita somente com $T_{3}, T_{4}$ e $T_{5}$. Pode-se dizer que os três níveis não se diferenciam na dose de $4 \mathrm{ml}(20 \mathrm{mg})$ (valor $p=0,37)$, que se diferenciam nas outras duas doses, $\mathrm{com}_{4}$ mais freqüente [valor $\mathrm{p}<0,00002$ com $3 \mathrm{ml}(15 \mathrm{mg})$ e valor $\mathrm{p}<0,000001$ em $2,5 \mathrm{ml}$ $(12,5 \mathrm{mg})$ ]. A moda foi $\mathrm{T}_{4}$ independente da dose utilizada (Tabela II).

Tabela II - Tempo de Latência, Dispersão Cefálica da Analgesia, Bloqueio Motor, Relaxamento Muscular, Complementação da Anestesia Geral e Falhas

\begin{tabular}{|c|c|c|c|}
\hline & $\begin{array}{c}20 \mathrm{mg} \\
(\mathrm{n}=20)\end{array}$ & $\begin{array}{c}15 \mathrm{mg} \\
(\mathrm{n}=40)\end{array}$ & $\begin{array}{l}12,5 \mathrm{mg} \\
(\mathrm{n}=40)\end{array}$ \\
\hline Tempo de latência * & $1,54 \pm 0,55$ & $1,53 \pm 0,64$ & $2,03 \pm 0,98$ \\
\hline \multicolumn{4}{|l|}{ Nível sensitivo } \\
\hline $\mathrm{T}_{3}$ & 6 & 0 & 0 \\
\hline $\mathrm{T}_{4}$ & 9 & 23 & 33 \\
\hline $\mathrm{T}_{5}$ & 4 & 13 & 4 \\
\hline $\mathrm{T}_{6}$ & 1 & 2 & 0 \\
\hline $\mathrm{T}_{7}$ & 0 & 0 & 1 \\
\hline $\mathrm{T}_{8}$ & 0 & 0 & 2 \\
\hline $\mathrm{T}_{9}$ & 0 & 0 & 0 \\
\hline $\mathrm{T}_{10}$ & 0 & 0 & 0 \\
\hline$T_{11}$ & 0 & 1 & 0 \\
\hline Moda & $\mathrm{T}_{4}$ & $\mathrm{~T}_{4}$ & $\mathrm{~T}_{4}$ \\
\hline \multicolumn{4}{|l|}{ Bloqueio motor } \\
\hline 3 & 19 & 38 & 38 \\
\hline 2 & 01 & 01 & 02 \\
\hline 1 & - & - & - \\
\hline 0 & - & - & - \\
\hline \multicolumn{4}{|l|}{ Relaxamento muscular } \\
\hline Excelente & 19 & 38 & 37 \\
\hline Satisfatório & 01 & 01 & 01 \\
\hline Insatisfatório & - & - & 02 \\
\hline Falha & - & 01 & - \\
\hline Anestesia geral & - & 01 & 02 \\
\hline Falhas & - & 01 & - \\
\hline Volume infundido $(\mathrm{ml})$ * & $1492 \pm 290$ & $1583 \pm 350$ & $1575 \pm 464$ \\
\hline Tempo cirúrgico (h) * & $1,18 \pm 0,34$ & $1,18 \pm 0,39$ & $1,14 \pm 0,45$ \\
\hline
\end{tabular}

* Valores expressos em Média \pm DP. Há diferença significativa entre os tempos médios de latência (valor $\mathrm{p}=0,010$ ), com $20 \mathrm{mg}=15 \mathrm{mg}<12,5 \mathrm{mg}$ para $\alpha=0,05$

O bloqueio motor completo ocorreu em 95 pacientes sem diferença significativa entre os grupos. Pode-se afirmar que o bloqueio motor completo tem a mesma chance de ocorrer com as três doses utilizadas (valor $p=1,000$ )

O relaxamento muscular avaliado pelo cirurgião ocorreu em 94 pacientes sem diferença significativa entre os grupos (valor $p=0,99$ ) (Tabela II). Não houve necessidade de complementação da anestesia com $4 \mathrm{ml}(20 \mathrm{mg})$. A anestesia geral foi necessária em 1 paciente com $3 \mathrm{ml}$ (15 mg) e 2 pacientes com 2,5 ml (12,5 mg).

Não houve diferença significativa nos três grupos em relação ao volume médio infundido da solução de Ringer com lactato (Tabela II). O volume da solução de Ringer com lactato infundido variou de 1000 a $1500 \mathrm{ml}$. O tempo cirúrgico foi praticamente o mesmo independentemente da dose utilizada (Tabela II).

A média das pressões arteriais sistólica e diastólica está na figura 1. Utilizando o teste t-pareado, não foi observada diferença significativa quando se compararam as pressões arteriais nos momentos de enfermaria e de sala de operações e após bloqueio com as três doses de bupivacaína. Não houve necessidade do uso de vasopressor em nenhuma paciente.

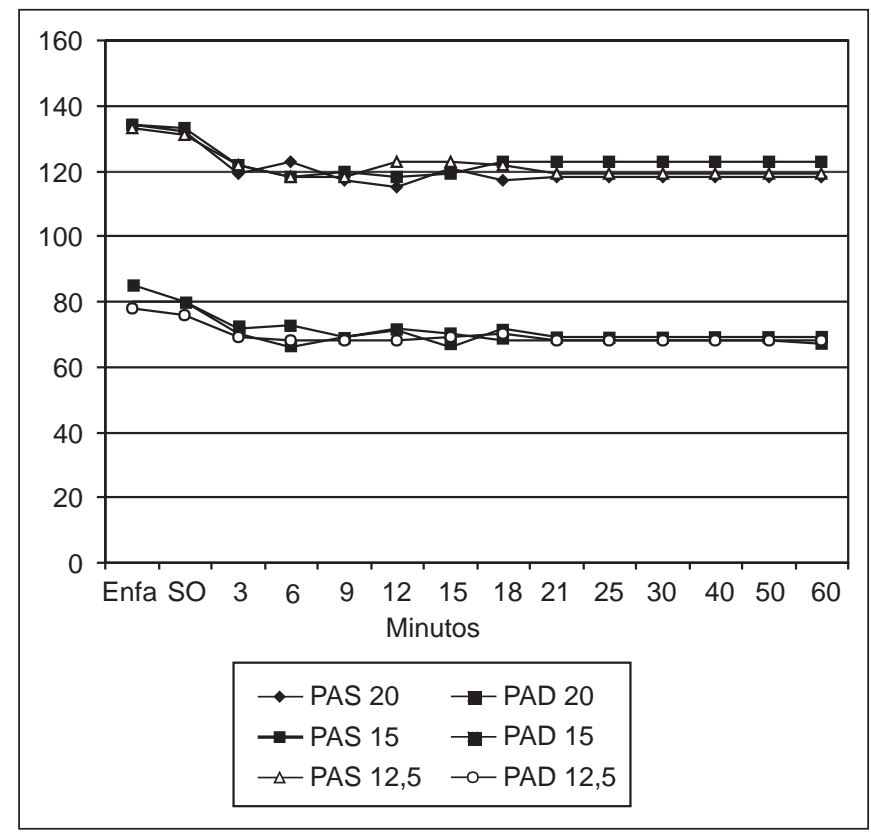

Figura 1- Avaliação da Pressão Arterial Sistólica e Diastólica nos Diferentes Grupos e nos Diferentes Tempos

Os valores do escore de Apgar estão na tabela III. No grupo $12,5 \mathrm{mg}$ houve dois fetos prematuros, com Apgar abaixo de 5 no primeiro minuto. No quinto minuto, todos os conceptos tiveram Apgar acima de 8.

Tabela III - Dados dos Recém-Nascidos

\begin{tabular}{lcccc}
\hline & $\begin{array}{c}20 \mathrm{mg} \\
(\mathrm{n}=20)\end{array}$ & $\begin{array}{c}15 \mathrm{mg} \\
(\mathrm{n}=40)\end{array}$ & $\begin{array}{c}12,5 \mathrm{mg} \\
(\mathrm{n}=40)\end{array}$ \\
\hline $\begin{array}{l}\text { Apgar mediano } \\
\text { (extremos) }\end{array}$ & $1 \mathrm{~min}$ & $8,95 \pm 0,22$ & $9,00 \pm 0,39$ & $8,37 \pm 1,67$ \\
& $5 \mathrm{~min}$ & $9,95 \pm 0,22$ & $9,94 \pm 0,22$ & $9,72 \pm 1,10$ \\
Apgar $<7$ & $1 \mathrm{~min}$ & 0 & 0 & 2 \\
& $5 \mathrm{~min}$ & 0 & 0 & 0 \\
\hline
\end{tabular}




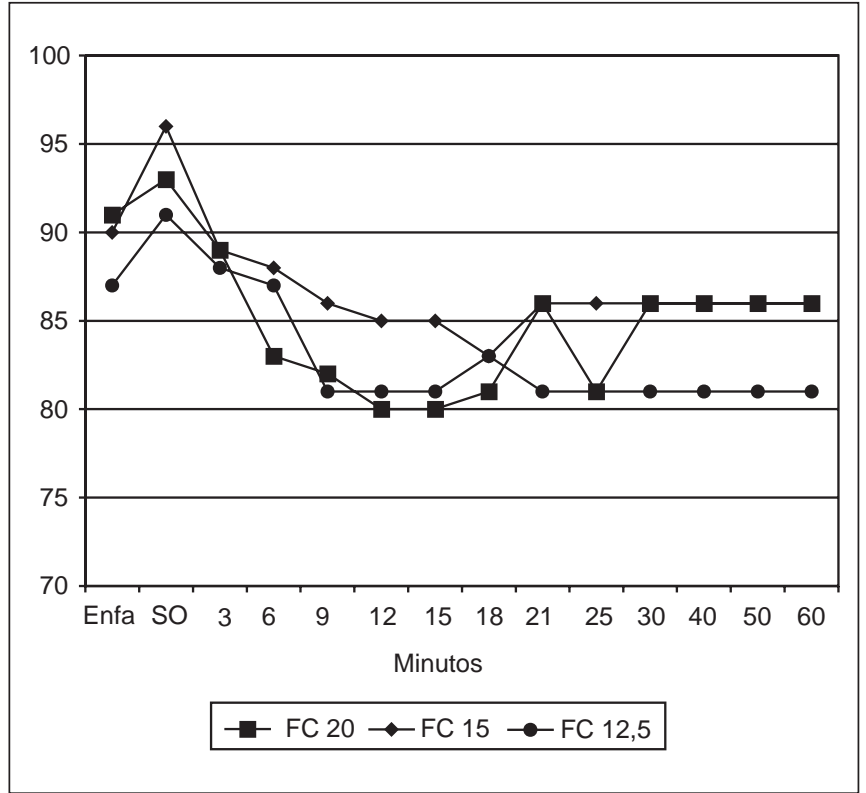

Figura 2 - Avaliação da Freqüência Cardíaca nos Diferentes Grupos e nos Diferentes Tempos

Náuseas e vômitos foram observados em apenas um paciente do grupo $15 \mathrm{mg}$. Ocorreu sonolência em duas pacientes com $3 \mathrm{ml}$ (20 mg), três com $3 \mathrm{ml}$ (15 mg) e nenhuma com 2,5 $\mathrm{ml}(12,5 \mathrm{mg})$. Dificuldade respiratória foi observada em duas pacientes com $4 \mathrm{ml}(20 \mathrm{mg}$ ) e nenhuma com $3 \mathrm{ml}$ ( $15 \mathrm{mg})$ e 2,5 $\mathrm{ml}(12,5 \mathrm{mg})$. Não foi possível comparar os três grupos por baixa freqüência de náuseas, vômitos, sonolência, dificuldade respiratória e vasopressor (Tabela IV).

Tabela IV - Náuseas e Vômitos, Sonolência, Dificuldade Respiratória e Vasopressor

\begin{tabular}{lccc}
\hline & $\begin{array}{c}20 \mathrm{mg} \\
(\mathrm{n}=20)\end{array}$ & $\begin{array}{c}15 \mathrm{mg} \\
(\mathrm{n}=40)\end{array}$ & $\begin{array}{c}12,5 \mathrm{mg} \\
(\mathrm{n}=40)\end{array}$ \\
\hline Náusea e vômito & - & 01 & - \\
Sonolência & 02 & 03 & - \\
Dificuldade respiratória & 02 & - & - \\
Vasopressor & - & - & - \\
\hline
\end{tabular}

Comparações 2 a 2 pelo teste Exato de Fisher

Apenas uma paciente desenvolveu cefaléia pós-punção, no grupo $3 \mathrm{ml}(15 \mathrm{mg})$ e a agulha utilizada foi a 27G. Acefaléia foi tratada com tiapridal e analgésico antitérmico.

\section{DISCUSSÃO}

O objetivo deste estudo foi avaliar a eficácia e segurança da raquianestesia com bupivacaína isobárica a $0,5 \%$ em diferentes volumes para cesarianas eletivas puncionadas em decúbito lateral esquerdo. Os volumes de 4, 3 e 2,5 ml produziram anestesia segura, sem hipotensão arterial, com baixa incidência de bradicardia, náuseas e vômitos.
A dispersão da bupivacaína isobárica pode ser variável, dependendo do volume e velocidade de injeção. A bupivacaína a $0,5 \%$ pura (baricidade a $37^{\circ} \mathrm{C}$ é de 0,9997$)^{7}$ pode se tornar ligeiramente hipobárica na temperatura do corpo. Este fato pode ser desprezado no presente trabalho devido à punção subaracnóidea ter sido realizada em decúbito lateral em todas as pacientes. A dose ideal de bupivacaína isobárica a $0,5 \%$ injetada em decúbito lateral ainda é desconhecida. Estudando 12,5 mg de bupivacaína a $0,5 \%$ isobárica não foi observada diferença no início de ação, na dispersão máxima da analgesia e na duração do bloqueio motor quando comparada com a mesma dose da solução hiperbárica ${ }^{8}$. Entretanto, a dose de $12,5 \mathrm{mg}$ refletiu numa maior necessidade de complementação com anestesia geral pelo relaxamento muscular insatisfatório. A similaridade entre a dispersão da solução isobárica com a hiperbárica de bupivacaína quando injetadas em decúbito lateral mostrou que a gravidez pode ter influência nesta dispersão ${ }^{8}$. No presente estudo com a adição de fentanil e morfina, em seringas separadas e a velocidade de injeção padronizada, a dispersão com as três doses foi semelhante.

A hipotensão arterial e a bradicardia são os efeitos colaterais mais temidos na raquianestesia ${ }^{9,10}$. Em obstetrícia as causas são multifatoriais, como alto nível de bloqueio simpático, compressão aortocava e bloqueio das fibras cardioaceleradoras. O tratamento pronto e eficaz é considerado fundamental para prevenir o sofrimento fetal. Admitindo que todas as medidas profiláticas convencionais, tais como expansão volêmica e deslocamento do útero, tenham sido adotadas, o uso profilático de vasopressores, notadamente da efedrina, tem sido sugerido como vantajoso tanto para a mãe como para o feto ${ }^{11,12}$. Por estar estudando soluções que sabidamente proporcionam menor incidência de hipotensão arterial ${ }^{13}$, o critério de hipotensão arterial usado foi o da diminuição de $30 \%$ da pressão arterial sistólica. Diferentemente de outros autores, não utilizamos o vasopressor profilaticamente nem por via muscular ${ }^{8}$ nem por via venosa ${ }^{11,12}$, para realmente avaliar a incidência de hipotensão arterial com a bupivacaína a $0,5 \%$ isobárica em diferentes volumes. Nas 100 pacientes pesquisadas não foi observado nenhum caso de hipotensão arterial, mostrando que a reposição em torno de $1000 \mathrm{ml}$ de solução de Ringer com lactado, o desvio do útero para esquerda e o aumento da concentração de oxigênio inspirado foram medidas suficientes para evitar a hipotensão arterial.

Vários artigos discutem a melhor forma de prevenir ou tratar a hipotensão arterial após raquianestesia para cesariana. $O$ uso profilático de glicopirrolato e a temperatura dos líquidos pré-infundidos não tem nenhuma correlação ${ }^{14,15}$. Em contraste, a velocidade de injeção das drogas na raquianestesia tem uma correlação significativa com a incidência e gravidade da hipotensão arterial ${ }^{16}$. Se as drogas são injetadas lentamente, resultam numa menor incidência de hipotensão arterial. Este fato confirma que a injeção lenta de opióides (em seringas separadas) e posteriormente da bupivacaína a $0,5 \%$ isobárica cursou com uma grande estabilidade cardiocirculatória na raquianestesia para cesarianas. 
Os opióides foram utilizados primeiramente para analgesia pós-operatória ${ }^{17}$. Os opióides lipofílicos têm um rápido início de ação (minutos) e curta duração de ação (1-4 h) com pequeno risco de depressão respiratória ${ }^{18}$. A adição de fentanil à raquianestesia produz uma analgesia sinérgica para a dor somática e visceral sem aumentar o bloqueio simpático ${ }^{19}$. A mistura de fentanil com o anestésico local pode diminuir a baricidade e alterar a distribuição dos agentes no $\mathrm{LCR}^{20}$, e a melhor relação risco benefício é a adição de 10 a $25 \mu \mathrm{g}$ de fentanil. Desta forma, o fentanil foi administrado na dose de 25 $\mu \mathrm{g}$ em seringa separada, para proporcionar sinergismo sem alterar a baricidade do anestésico local. A dose de $50 \mu \mathrm{g}$ de morfina foi usada para analgesia pós-operatória e da mesma forma em seringa separada.

Três pacientes desse estudo necessitaram complementação com anestesia geral (propofol e fentanil). A causa mais comum de dor durante cesariana é o nível inadequado de bloqueio sensitivo, unilateral ou abaixo de $\mathrm{T}_{5}$, dor visceral ou dor nos ombros ${ }^{21}$. Com $20 \mathrm{mg}$ todas as pacientes (100\%) apresentaram um bloqueio que variou de $\mathrm{T}_{3}-\mathrm{T}_{6}$, não havendo necessidade de complementação com anestesia geral. Entretanto, $30 \%$ das pacientes apresentaram bloqueio sensitivo em $\mathrm{T}_{3}$, resultando em dificuldade respiratória em $10 \%$ das pacientes. Com $15 \mathrm{mg}$, trinta e oito pacientes (95\%) apresentaram bloqueio sensitivo entre $T_{4}-T_{6}$, resultando na necessidade de complementação em uma paciente. Com 12,5 mg, trinta e sete $(92,5 \%)$ apresentaram bloqueio sensitivo entre $\mathrm{T}_{4}-\mathrm{T}_{6}$, resultando na necessidade de complementação em duas pacientes. Este trabalho mostrou que o aumento da dose resulta numa melhor qualidade do bloqueio com melhor relaxamento muscular abdominal.

Diversos métodos têm sido propostos para prevenir o aparecimento de náuseas e vômitos durante raquianestesia em cesariana. Doses sub-hipnóticas contínua de propofol ${ }^{22} \mathrm{e}$ fentanil subaracnóideo ${ }^{23}$ têm sido indicadas. A utilização de efedrina em infusão ou bolus não foi suficiente para diminuir a incidência de náuseas (15\% a 50\%) e vômitos (5\% a 15\%)

${ }^{11,12}$ quando se utilizou bupivacaína a $0,5 \%$ hiperbárica. Com a bupivacaína isobárica associada ao fentanil, a incidência de náuseas e vômitos foi muito baixa (1\%), mostrando que esta associação melhora a qualidade da anestesia e resulta numa menor incidência de efeitos colaterais, como hipotensão arterial, náuseas e vômitos.

Em 1986, usando termografia, foi demonstrado que as soluções de lidocaína e tetracaína hiperbáricas produzem um bloqueio simpático de até seis segmentos acima do nível de analgesia ${ }^{24}$. Estudos com soluções puras ainda não foram realizados; portanto, não podemos afirmar que não ocorre uma dissociação entre os bloqueios sensitivo e simpático.

Em conclusão, este estudo mostrou que a bupivacaína a $0,5 \%$ isobárica nos volumes de 4,3 e 2,5 ml, quando injetada em decúbito lateral, proporcionou uma rápida e segura anestesia para cesariana. Esta solução promoveu grande estabilidade cardiocirculatória, sem necessidade do uso de vasopressor e baixa incidência de efeitos colaterais. Estudos da relação entre os bloqueios sensitivo e simpático precisam ser realizados com as soluções isobáricas.

\section{Spinal Anesthesia for Cesarean Section with $0.5 \%$ Isobaric Bupivacaine plus Fentanyl and Morphine. Prospective Study with Different Volumes}

Luiz Eduardo Imbelloni, TSA, M.D., Eneida Maria Vieira, M.D., Ana Rocha, M.D., Marildo Assunção Gouveia, TSA, M.D., José Antônio Cordeiro, M.D.

\section{INTRODUCTION}

Spinal anesthesia has been more widely used than epidural anesthesia for cesarean section ${ }^{1}$ because of some advantages, such as: simpler spinal space identification, lower local anesthetic dose, lower cost and faster onset with consequent shorter operating room stay ${ }^{2-4}$.

The introduction of finer needles in the early 1990s has revived the interest on spinal anesthesia for all procedures compatible with the technique. Spinal anesthesia for cesarean section with $0.5 \%$ hyperbaric bupivacaine is related to $37.5 \%$ arterial hypotension ${ }^{2}$ with early onset ${ }^{2-4}$. Based on a study published in $1988^{5}, 0.5 \%$ isobaric bupivacaine was counterindicated for obstetric spinal anesthesia when the puncture was performed in the sitting position ${ }^{6}$.

There is no publication in the Brazilian literature on $0.5 \%$ isobaric bupivacaine for cesarean sections. This prospective study aimed at evaluating $0.5 \%$ isobaric bupivacaine plus fentanyl or morphine in separate syringes, administered with the patients in the lateral position, to correlate the incidence of hemodynamic complications and the cephalad spread with different volumes.

\section{METHODS}

After the Hospital's Ethics Committee approval and their formal consent, participated in this prospective study 100 patients aged 15 years or above and less than 40 years, weight 50 to $120 \mathrm{~kg}$ and height 145 to $175 \mathrm{~cm}$, scheduled for elective cesarean sections. Patients with obstetric complications were not included in the study. Exclusion criteria were: hypovolemia, coagulation abnormalities, infection and refusal of the proposed method. Patients were randomly allocated in three groups to receive $4 \mathrm{ml}(20 \mathrm{mg}), 3 \mathrm{ml}$ (15 mg) or $2.5 \mathrm{ml}$ (12.5 mg) $0.5 \%$ isobaric bupivacaine.

Patients were not premedicated. After venoclysis with an $18 \mathrm{G}$ catheter, lactated Ringer's was infused. There has been no pre volume expansion and the volume infused was checked at surgery completion.

Spinal puncture was performed with the patient in the lateral position in the median or paramedian line at $L_{3}-L_{4}$ interspace 
with a 27G Quincke needle without introducer. After CSF reflux through the needle's gun and with the bevel in the cephalad direction, $25 \mu \mathrm{g}$ fentanyl and $50 \mu \mathrm{g}$ morphine were previously injected through separate insulin syringes, followed by $0.5 \%$ isobaric bupivacaine according to the studied group at a rate of $1 \mathrm{ml} .15 \mathrm{sec}^{-1}$, without barbotage. After injection, patients were placed in the supine position with the uterus displaced to the left.

Patients heart rate and blood pressure were evaluated by automatic and non-invasive method. Values obtained in the room were considered baseline. Blood pressure and heart rate were evaluated before spinal puncture, at 3-minute intervals until 21 minutes and then at 5-minute intervals until surgery completion. Oxygen saturation was continuously measured by pulse oximetry. ECG was continuously measured at CM5. Arterial hypotension, defined as SBP decrease above $30 \%$ of baseline was treated with $10 \mathrm{mg}$ intravenous ephedrine. Heart rate below $60 \mathrm{bpm}$ would be treated with atropine, and nausea and vomiting would be treated with $20 \mathrm{mg}$ intravenous metoclopramide. If there were pain or discomfort during surgery $50 \mu \mathrm{g}$ bolus fentanyl and propofol would be administered. Oxygen via nasal catheter was administered at a 2 L. min $^{-1}$ flow throughout the surgery. Neonates were evaluated by Apgar score at one and five minutes of life. Midazolam ( 1 to $3 \mathrm{mg}$ ) was only administered after fetal extraction. Surgery duration was recorded.

Blockade onset was defined as time for the first loss of sensitivity in the region corresponding to puncture metamer. Segmental anesthesia level (loss of sensation at pinprick) was bilaterally determined at 1 -minute intervals in the beginning and at 5-minute intervals until 20 minutes were elapsed. Lower limbs motor block was evaluated at the same intervals using Bromage's modified scale.

Abdominal relaxation quality was evaluated by the surgeon as excellent (with muscle relaxation), satisfactory (acceptable muscle relaxation) and unsatisfactory (no muscle relaxation). Vesical catheter was used. The incidence of headache was evaluated for all patients until the $5^{\text {th }}$ postoperative day.

Data are expressed in mean \pm SD, median or frequency (\%), when appropriate. Statistical tools were Chi-square with minimum weighted squares, Fisher Exact test, Analysis of Variance (ANOVA) with Tukey's paired comparisons, univariate Student's $t$ test for paired data, being considered significant $p<0.05$.

\section{RESULTS}

Demographics data and studied population are shown in table I. Due to results obtained with $3 \mathrm{ml}(15 \mathrm{mg})$ and $2.5 \mathrm{ml}(12.5$ $\mathrm{mg}$ ), the $4 \mathrm{ml}$ (20 mg) group was closed with 20 patients. There has been one spinal anesthesia failure in the $3 \mathrm{ml}$ group.

Analgesic onset was fast with all doses. Onset was significantly longer with the lowest dose (Table II). Analgesic cephalad spread is shown in table II. The low incidence of sensory level below $T_{5}$ allowed the statistical analysis to be performed only with $T_{3}, T_{4}$ and $T_{5}$. One may say that the three levels did not differ for the $4 \mathrm{ml}(20 \mathrm{mg})$ dose $(p=0.37)$, but did differ for the other doses, with more frequent $T_{4}[p<0.00002$ with $3 \mathrm{ml}(15 \mathrm{mg})$ and $\mathrm{p}<0.000001$ with $2.5 \mathrm{ml}(12.5 \mathrm{mg})]$. Mode was $\mathrm{T}_{4}$ regardless of the dose (Table II).

Table I - Demographics Data (Mean \pm SD)

\begin{tabular}{lcccc}
\hline & $\begin{array}{c}20 \mathrm{mg} \\
(\mathrm{n}=20)\end{array}$ & $\begin{array}{c}15 \mathrm{mg} \\
(\mathrm{n}=40)\end{array}$ & $\begin{array}{c}12.5 \mathrm{mg} \\
(\mathrm{n}=40)\end{array}$ & p value \\
\hline Age (years) & $27.10 \pm 7.27$ & $24.75 \pm 6.13$ & $26.82 \pm 6.02$ & 0.25 \\
Weight $(\mathrm{kg})$ & $68.25 \pm 8.46$ & $74.55 \pm 10.98$ & $75.97 \pm 14.03$ & 0.06 \\
Height $(\mathrm{cm})$ & $157.90 \pm 5.29$ & $162.20 \pm 6.17$ & $162 \pm 6.11$ & $0.02^{*}$ \\
\hline$*$
\end{tabular}

${ }^{*} p<0.05$

Table II - Analgesia Onset and Cephalad Spread, Motor Block, Muscle Relaxation, General Anesthesia Complementation and Failures

\begin{tabular}{lccc}
\hline $\begin{array}{c}20 \mathrm{mg} \\
(\mathrm{n}=20)\end{array}$ & $\begin{array}{c}15 \mathrm{mg} \\
(\mathrm{n}=40)\end{array}$ & $\begin{array}{c}12.5 \mathrm{mg} \\
(\mathrm{n}=40)\end{array}$ \\
\hline Onset $^{*}$ & $1.54 \pm 0.55$ & $1.53 \pm 0.64$ & $2.03 \pm 0.98$
\end{tabular}

Sensory level

\begin{tabular}{|c|c|c|c|}
\hline $\mathrm{T}_{3}$ & 6 & 0 & 0 \\
\hline $\mathrm{T}_{4}$ & 9 & 23 & 33 \\
\hline$T_{5}$ & 4 & 13 & 4 \\
\hline $\mathrm{T}_{6}$ & 1 & 2 & 0 \\
\hline $\mathrm{T}_{7}$ & 0 & 0 & 1 \\
\hline $\mathrm{T}_{8}$ & 0 & 0 & 2 \\
\hline $\mathrm{T}_{9}$ & 0 & 0 & 0 \\
\hline $\mathrm{T}_{10}$ & 0 & 0 & 0 \\
\hline$T_{11}$ & 0 & 1 & 0 \\
\hline Mode & $\mathrm{T}_{4}$ & $\mathrm{~T}_{4}$ & $\mathrm{~T}_{4}$ \\
\hline \multicolumn{4}{|l|}{ Motor block } \\
\hline 3 & 19 & 38 & 38 \\
\hline 2 & 01 & 01 & 02 \\
\hline 1 & - & - & - \\
\hline 0 & - & - & - \\
\hline \multicolumn{4}{|l|}{ Muscle relaxation } \\
\hline Excellent & 19 & 38 & 37 \\
\hline Satisfactory & 01 & 01 & 01 \\
\hline Unsatisfactory & - & - & 02 \\
\hline Failure & - & 01 & - \\
\hline General anesthesia & - & 01 & 02 \\
\hline Failures & - & 01 & - \\
\hline Infused volume $(\mathrm{ml})$ * & $1492 \pm 290$ & $1583 \pm 350$ & $1575 \pm 464$ \\
\hline Surgery duration $(\mathrm{h})$ * & $1.18 \pm 0.34$ & $1.18 \pm 0.39$ & $1.14 \pm 0.45$ \\
\hline
\end{tabular}

* Values expressed in Mean \pm SD. There is significant difference between mean onset $(p=0.010)$, with $20 \mathrm{mg}=15 \mathrm{mg}<12.5 \mathrm{mg}$ for $\alpha=0.05$

Total motor block was observed in 95 patients without statistical difference among groups. One may state that there is the same chance for total motor block with the three doses $(p=$ 1.000).

Muscle relaxation evaluated by the surgeon was observed in 94 patients without statistical difference among groups ( $p=$ 
0.99). There was no need for anesthetic complementation with $4 \mathrm{ml}(20 \mathrm{mg})$. General anesthesia was needed for one 3 $\mathrm{ml}(15 \mathrm{mg})$ patient and two $2.5 \mathrm{ml}(12.5 \mathrm{mg})$ patients.

There has been no significant difference among groups in mean lactated Ringer's solution volume infused (Table II). Infused lactated Ringer's volume has varied from 1000 to 1500 $\mathrm{ml}$. Surgery duration was virtually the same regardless of the dose used (Table II).

Mean systolic and diastolic blood pressure is shown in figure 1. Using tpaired test, there were no statistically significant differences when blood pressures in the ward and in the operating room and after blockade with the three bupivacaine doses were compared. No patient needed vasopressants.

Apgar score values are shown in table III. There were two premature fetuses in the $12.5 \mathrm{mg}$ group, with Apgar score below 5 in the first minute. At five minutes, all neonates had Apgar score above 8.

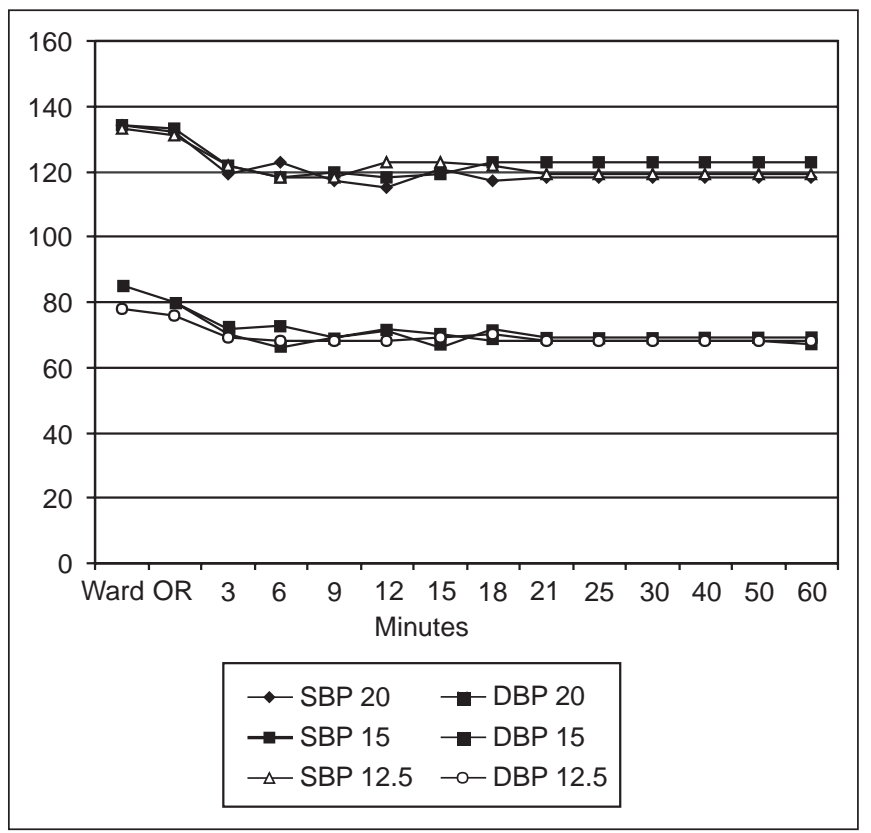

Figure 1 - Systolic and Diastolic Blood Pressure Evaluation in Different Groups and Different Times

Table III - Neonates Data

\begin{tabular}{lcccc}
\hline & $\begin{array}{c}20 \mathrm{mg} \\
(\mathrm{n}=20)\end{array}$ & $\begin{array}{c}15 \mathrm{mg} \\
(\mathrm{n}=40)\end{array}$ & $\begin{array}{c}12.5 \mathrm{mg} \\
(\mathrm{n}=40)\end{array}$ \\
\hline $\begin{array}{l}\text { Median Apgar } \\
\text { (extremes) }\end{array}$ & $1 \mathrm{~min}$ & $8.95 \pm 0.22$ & $9.00 \pm 0.39$ & $8.37 \pm 1.67$ \\
& $5 \mathrm{~min}$ & $9.95 \pm 0.22$ & $9.94 \pm 0.22$ & $9.72 \pm 1.10$ \\
Apgar $<7$ & $1 \mathrm{~min}$ & 0 & 0 & 2 \\
& $5 \mathrm{~min}$ & 0 & 0 & 0 \\
\hline
\end{tabular}

Nausea and vomiting were observed in one $15 \mathrm{mg}$ patient. There has been somnolence in two $4 \mathrm{ml}(20 \mathrm{mg})$ patients, three $3 \mathrm{ml}(15 \mathrm{mg})$ patients and no $2.5 \mathrm{ml}(12.5 \mathrm{mg})$ patient. Respiratory difficulties were observed in two $4 \mathrm{ml}(20 \mathrm{mg})$ pa-

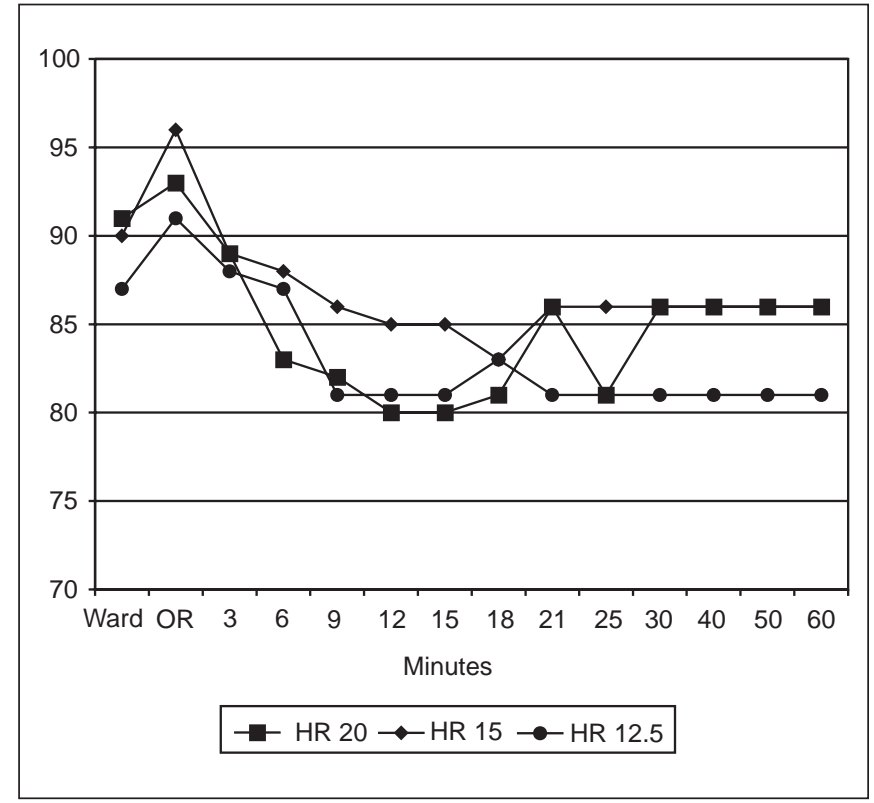

Figure 2 - Heart Rate Evaluation in Different Groups and Different Times

tients and no $3 \mathrm{ml}(15 \mathrm{mg})$ and $2.5 \mathrm{ml}(12.5 \mathrm{mg})$ patients. It was impossible to compared groups due to the low incidence of nausea, vomiting, somnolence, respiratory difficulty and vasopressant (Table IV).

Table IV - Nausea and Vomiting, Somnolence, Respiratory Difficulty and Vasopressant

\begin{tabular}{lccc}
\hline & $\begin{array}{c}20 \mathrm{mg} \\
(\mathrm{n}=20)\end{array}$ & $\begin{array}{c}15 \mathrm{mg} \\
(\mathrm{n}=40)\end{array}$ & $\begin{array}{c}12.5 \mathrm{mg} \\
(\mathrm{n}=40)\end{array}$ \\
\hline Nausea and vomiting & - & 01 & - \\
Somnolence & 02 & 03 & - \\
Respiratory difficulty & 02 & - & - \\
Vasopressant & - & - & - \\
\hline
\end{tabular}

Fisher's Exact test 2 by 2 comparisons

Just one $3 \mathrm{ml}$ ( $15 \mathrm{mg}$ ) patient developed post-dural puncture headache with a $27 \mathrm{G}$ needle. Headache was treated with thiapridal and antithermic analgesics.

\section{DISCUSSION}

This study aimed at evaluating spinal anesthesia safety and efficacy with $0.5 \%$ isobaric bupivacaine in different volumes for elective cesarean sections, induced in the left lateral position. Volumes of 4,3 and $2.5 \mathrm{ml}$ have produced safe anesthesia without arterial hypotension and with a low incidence of bradycardia, nausea and vomiting.

Isobaric bupivacaine spread may vary depending on the volume and infusion rate. Pure $0.5 \%$ bupivacaine (baricity at 37 ${ }^{\circ} \mathrm{C}$ is 0.9997$)^{7}$ may become slightly hypobaric at body temperature. This fact may be discarded in the present study be- 
cause spinal puncture was performed in the lateral position in all patients. The ideal $0.5 \%$ isobaric bupivacaine dose to be injected in the lateral position is still unknown. Studying 12.5 $\mathrm{ml}$ of $0.5 \%$ isobaric bupivacaine, no difference has been observed in onset, maximum analgesic spread and motor block duration as compared to the same hyperbaric dose ${ }^{8}$. However, $12.5 \mathrm{ml}$ has reflected a higher need for general anesthesia complementation due to unsatisfactory muscle relaxation. The similarity between isobaric and hyperbaric bupivacaine spread when injected in the lateral position has shown that pregnancy may influence this spread ${ }^{8}$. In our study, with the association of fentanyl and morphine in separate syringes and standardized injection rate, spread was similar with the three doses.

Arterial hypotension and bradycardia are the most feared spinal anesthesia side-effects ${ }^{9,10}$. In obstetrics, they have multifactorial causes such as high sympathetic block level, aorta-cava compression and cardioaccelerator fibers block. Prompt and effective treatment is critical to prevent fetal suffering. Assuming that all conventional prophylactic measures, such as volume expansion and uterus displacement were adopted, the prophylactic use of vasopressants, especially ephedrine, has been suggested as advantageous both for mother and the fetus ${ }^{11,12}$. For studying solutions which knowingly provide a lower incidence of arterial hypotension ${ }^{13}$, our criteria was $30 \%$ decrease in systolic blood pressure. Differently from other authors, we have not used prophylactic muscular ${ }^{8}$ or intravenous ${ }^{11,12}$ vasopressants to really evaluate the incidence of arterial hypotension with $0.5 \%$ isobaric bupivacaine in different volumes. There has been no arterial hypotension in all 100 patients evaluated showing that replacement with approximately 1000 ml lactated Ringer's, uterine displacement to the left and increased inspired oxygen concentration were enough to prevent arterial hypotension.

Several studies discuss the best way to prevent or treat arterial hypotension following spinal anesthesia for cesarean section. Prophylactic glycopirrolate and pre-infused fluid temperature have no correlation ${ }^{14,15}$. Conversely, drug injection rate has a significant correlation with arterial hypotension incidence and severity ${ }^{16}$. Drugs slowly injected result in less arterial hypotension. This fact confirms that slow opioid injections (in separate syringes) followed by $0.5 \%$ isobaric bupivacaine have resulted in high cardiocirculatory stability during spinal anesthesia for cesarean section.

Opioids were firstly used for postoperative analgesia ${ }^{17}$. Lipophylic opioids have fast onset (minutes) and short duration (1-4h) with low risk for respiratory depression ${ }^{18}$. The association of fentanyl to spinal anesthesia produces a synergistic analgesia for somatic and visceral pain without increasing sympathetic block ${ }^{19}$. The association of fentanyl and local anesthetics may decrease baricity and change agents' distribution in the $\mathrm{CSF}^{20}$, and the best cost/benefit ratio is to add 10 to $25 \mu \mathrm{g}$ fentanyl. So, $25 \mu \mathrm{g}$ fentanyl were administered in separate syringes to provide synergism without changing local anesthetics baricity. Also in separate syringes, $50 \mu \mathrm{g}$ morphine were used for postoperative analgesia.
Three patients in our study needed general anesthesia complementation (propofol and fentanyl). The most common cause of pain during cesarean section is the inadequate sensory block level, unilateral or below $T_{5}$, visceral pain or shoulder pain ${ }^{21}$. With $20 \mathrm{mg}$, all patients $(100 \%)$ had blockades varying from $T_{3}-T_{6}$, with no need for general anesthesia complementation. However, $30 \%$ of patients had sensory block in $\mathrm{T}_{3}$, resulting in respiratory difficulties for $10 \%$ of patients. With $15 \mathrm{mg}, 38$ patients $(95 \%)$ had sensory block between $\mathrm{T}_{4}-\mathrm{T}_{6}$ resulting in the need for complementation in one patient. With $12.5 \mathrm{mg}, 37$ (92.5\%) had sensory block between $\mathrm{T}_{4}-\mathrm{T}_{6}$, resulting in the need for complementation in two patients. This study has shown that higher doses result in better blockade with better abdominal muscle relaxation.

Several methods have been proposed to prevent nausea and vomiting during spinal anesthesia for cesarean section. Continuous sub-hypnotic doses of spinal propofol ${ }^{22}$ and fentanyl ${ }^{23}$ have been indicated. Infusion or bolus ephedrine was not enough to decrease nausea (15\% to $50 \%$ ) and vomiting (5\% to $15 \%)^{11,12}$ when $0.5 \%$ hyperbaric bupivacaine was used. With isobaric bupivacaine associated to fentanyl, the incidence of nausea and vomiting was very low (1\%) showing that this association improves anesthesia quality resulting in a lower incidence of side-effects, such as arterial hypotension, nausea and vomiting.

Thermography, in 1986, has shown that hyperbaric lidocaine and tetracaine induce sympathetic block up to 6 segments above the level of analgesia ${ }^{24}$. To date, there are no studies with pure solutions, so it is impossible to state that there is a dissociation between sensory and sympathetic blocks.

In conclusion, our study has shown that $0.5 \%$ isobaric bupivacaine, in the volumes of 4,3 and $2.5 \mathrm{ml}$ injected in the lateral position, has provided fast and safe anesthesia for cesarean section. The solution has provided high cardiocirculatory stability with no need for vasopressants and a low incidence of side-effects. Further studies are needed on the relationship between sensory and sympathetic blocks with isobaric solutions.

\section{REFERÊNCIAS - REFERENCES}

01. Villar GCP, Rosa C, Cappelli EL et al - Incidência de cefaléia pós-raquianestesia em pacientes obstétricas com o uso de agulha de Whitacre calibre 27G. Experiência com 4570 casos. Rev Bras Anestesiol, 1999;49:110-112.

02. Neves JFNP, Monteiro GA, Almeida JR et al - Raquianestesia para cesariana: avaliação da cefaléia com agulhas de Quincke e Whitacre 25G e 27G. Rev Bras Anestesiol, 1999;49:173-175.

03. Imbelloni LE, Carneiro ANG, Sobral MGC - Complicações da anestesia subaracnóidea com bupivacaína hiperbárica para cesariana: estudo prospectivo. Rev Bras Anestesiol, 1996;46: 13-18.

04. Carvalho JCA, Siaulys MM, Kuriki W et al - Estudo comparativo de agulhas Quincke vs Whitacre, calibre $5(25 \mathrm{G}) \mathrm{em}$ raquianestesia para cesárea. Rev Bras Anestesiol, 1993;43: 239-243. 
05. Carvalho JCA, Mathias RS, Torres MLA et al - Effect of baricity on subarachnoid anesthesia with bupivacaine for cesarean section. Reg Anesth, 1988;13:(Supp 1S):14.

06. Carvalho JCA, Mathias RS - Raquianestesia em Obstetrícia, em: Imbelloni LE - Raquianestesia, Colina-Revinter, 1995; 73-86.

07. Imbelloni LE, Beato L - Comparação entre bupivacaína racêmica (S50-R50) e mistura enantiomérica de bupivacaína (S75-R25), ambas isobáricas, a $0,5 \%$ em raquianestesia. Estudo em cirurgias ortopédicas. Rev Bras Anestesiol, 2001; 51:369-376

08. Russell IF, Holmqvist LO - Subarachnoid analgesia for caesarian section. A double-blind comparison of plain and hyperbaric 0.5\% bupivacaine. Br J Anaesth, 1987;59:347-353.

09. Carpenter RL, Caplan RA, Brown DL et al - Incidence and risk factors for side effects of spinal anesthesia. Anesthesiology, 1992;76:906-916.

10. Auroy $\mathrm{Y}$, Naschi P, Messiah A et al - Severe complications related to regional anesthesia: Results of a prospective survey in France. Anesthesiology, 1997;87:479-486.

11. Carvalho JCA, Cardoso MMSC, Capelli EL et al - Efedrina profilática durante raquianestesia para cesariana: estudo dose-resposta da administração em bolus e em infusão contínua. Rev Bras Anestesiol, 1999;49:309-314.

12. Carvalho JCA, Cardoso MMSC, Lorenz E et al - Efedrina profilática durante raquianestesia para cesariana: bolus seguido de infusão contínua em dose fixa e infusão contínua de dose decrescentes. Rev Bras Anestesiol, 2000;50:425-430.

13. Imbelloni LE - O Uso Racional da Raquianestesia, em: Imbelloni LE - Tratado de Anestesia Raquidiana, Curitiba, 2001;8:74-86.

14. Yentis SM, Jenkins CS, Lucas DN et al - The effect of prophylactic glycopyrrolate on maternal haemodynamics following spinal anaesthesia for elective caesarean section. Int J Obstet Anesth, 2000;9:156-159.

15. Jorgensen HS, Bach LF, Helbo-Hansen HS et al - Warm or cold saline for volume preload before spinal anaesthesia for caesarean section? Int J Obstet Anesth, 2000;9:20-25.

16. Simon L, Boulay G, Ziane AF et al - Effect of injection rate on hypotension associated with spinal anesthesia for cesarean section. Int J Obstet Anesth, 2000;9:10-14.

17. Imbelloni LE - Morfina peridural: avaliação da analgesia e força expiratória no pós-operatório imediato. Rev Bras Anestesiol, 1982;32:25-31.

18. Reuben SS, Dunn SM, Duprat KM et al - An intrathecal fentanyl dose-response study in lower extremity revascularization procedures. Anesthesiology, 1994;81:1371-1375

19. Hamber EA, Viscomi CM - Intrathecal lipophilic opioids as adjuncts to surgical spinal anesthesia. Reg Anesth Pain Med, 1999;24:255-263.

20. Parlow JL, Moneu P, Chan PS et al - Addition of opioids alters the density and spread of intrathecal local anesthetics? An in vitro study. Can J Anaesth, 1999;46:66-70.

21. Capogna G, Celleno D - Improving epidural anesthesia during cesarean section: causes of maternal discomfort or pain during surgery. Int J Obstet Anesth, 1994;3:149-152.
22. Numazaki M, Fujii Y - Subhypnotic dose of propofol for prevention of nausea and vomiting during spinal anaesthesia for caesarean section. Anaesth Intensive Care, 2000;28:262-265.

23. Manullang TR, Viscomi CM, Pace NL - Intrathecal fentanyl is superior to intravenous ondansetron for the prevention of perioperative nausea during cesarean delivery with spinal anesthesia. Anesth Analg, 2000;90:1162-1166.

24. Chamberlain DP, Chamberlain BDL - Changes in skin temperature of the trunk and their relationship with sympathetic blockade during spinal anesthesia. Anesthesiology, 1986;65: 139-143.

\section{RESUMEN}

Imbelloni LE, Vieira EM, Rocha A, Gouveia MA, Cordeiro JA Raquianestesia para Cesariana con Bupivacaína a 0,5\% Isobárica Asociada al Fentanil y Morfina. Estudio Prospectivo con Diferentes Volúmenes

JUSTIFICATIVA Y OBJETIVOS: La raquianestesia para cesariana fue descrita pocos años después del primer relato de Bier en 1898 y en los últimos 5 años ella se volvió método de elección en nuestro hospital. El objetivo de este estudio prospectivo en cesariana es evaluar el uso de la bupivacaína a $0,5 \%$ isobárica administrada con las parturientas en decúbito lateral, después de inyección de fentanil y morfina, y correlacionar con la incidencia de complicaciones hemodinámicas y dispersión cefálica con diferentes volúmenes.

MÉTODO: Cien pacientes sometidas a raquianestesia para cesariana electiva fueron aleatoriamente separadas en tres grupos que recibieron: $4 \mathrm{ml}(20 \mathrm{mg}), 3 \mathrm{ml}(15 \mathrm{mg})$ y 2,5 $\mathrm{ml}(12,5$ $\mathrm{mg}$ ) de bupivacaína a 0,5\% isobárica añadida de $25 \mu \mathrm{g}$ de fentanil y $50 \mu \mathrm{g}$ de morfina inyectados antes del anestésico. Fueron evaluados y comparados los siguientes parámetros: latencia de la analgesia, bloqueo motor, dispersión cefálica de la analgesia, alteraciones cardiovasculares e incidencia de náuseas y vómitos.

RESULTADOS: Los tres volúmenes de bupivacaína a 0,5\% isobárica produjeron efectos comparables. El tiempo de latencia fue mayor con la menor dosis. No fueron observadas diferencias en la dispersión cefálica, en el número de pacientes que tuvieron niveles cervicales, en las alteraciones cardiovasculares y en la incidencia de cefalea pós-punción. El nivel máximo de la analgesia fue $T_{4}$ (amplitud: $T_{3}-T_{6}$ ) con $4 \mathrm{ml}$, $T_{4}$ (amplitud: $T_{4}-T_{11}$ ) con $3 \mathrm{ml}$ y $T_{4}$ (amplitud: $T_{4}-T_{8}$ ) con $2,5 \mathrm{ml}$. Ninguna paciente necesitó de efedrina para tratar hipotensión arterial. EL bloqueo motor no fue completo en todas las pacientes. Una paciente desenvolvió cefalea pós-punción.

CONCLUSIONES: El resultado de este estudio confirma que la bupivacaína a $0,5 \%$ isobárica inyectada después de administración en jeringas separadas de fentanil y morfina y en decúbito lateral en los volúmenes de 2,5, 3 y $4 \mathrm{ml}$ proporciona una rápida y efectiva anestesia para cesariana. 